UDC 512.543 .7

\title{
HOW TO GENERALIZE THE KNOWN RESULTS ON EQUATIONS OVER GROUPS
}

\author{
Anton A. Klyachko
}

Faculty of mechanics and mathematics, Moscow State University.

Moscow 119992, Leninskie gory, MSU.

klyachko@daniil.math.msu.su

\begin{abstract}
The known facts about solvability of equations over groups are considered from a more general point of view. A generalized version of the theorem about solvability of unimodular equations over torsion-free groups is proved. In a special case, this generalized version become a multivariable variant of this theorem. For unimodular equations over torsion free groups, we prove an analogue of Magnus's Freiheitssatz, which asserts that there exists a solution with good behavior with respect to free factors of the initial group.

Key words: equations over groups, Kervaire-Laudenbach conjecture, Freiheitssatz.
\end{abstract}

\section{Introduction}

An equation over a group $G$ with unknown (or variable) $t$ is a formal expression of the form

$$
g_{1} t^{\varepsilon_{1}} g_{2} t^{\varepsilon_{2}} \ldots g_{n} t^{\varepsilon_{n}}=1
$$

where $g_{i} \in G, \varepsilon_{i} \in \mathbb{Z}$. Equation $(*)$ is called solvable over the group $G$ if there exist a group $\widetilde{G}$ containing $G$ as a subgroup and an element $\widetilde{t} \in \widetilde{G}$ (called a solution to equation $(*)$ ) such that $g_{1} \widetilde{t}^{\varepsilon_{1}} g_{2} \widetilde{t}^{\varepsilon_{2}} \ldots g_{n} \widetilde{t}^{\varepsilon_{n}}=1$ in $\widetilde{G}$.

There is a lot of theorems (see, e.g., [1]-[14], [18]), wich say that equation (*) is solvable under some conditions on the group $G$ the left-hand side of the equation.

In this paper, we suggest a generalization of the notion of equation over a group. A generalized equation over a group $G$ with a variable group $T$ is a formal expressio of the form

$$
g_{1} t_{1} g_{2} t_{2} \ldots g_{n} t_{n}=1
$$

where $g_{i} \in G, t_{i} \in T$. We call generalized equation $\left(*^{\prime}\right)$ solvable over the group $G$ if there exist a group $\widetilde{G}$ containing $G$ as a subgroup and a homomorphism $T \rightarrow \widetilde{G}, t \mapsto \widetilde{t}$ (called a solution to generalized equation $\left.\left(*^{\prime}\right)\right)$ such that $g_{1} \widetilde{t}_{1} g_{2} \widetilde{t}_{2} \ldots g_{n} \widetilde{t}_{n}=1$ in $\widetilde{G}$.

Clearly, in the case of infinite cyclic variable group, the notion of the solvability of a generalized equation coincides with the notion of the solvability of an ordinary equation. It is suggested to prove generalized analogues of known theorems on solvability of equations. Such generalized analogues may include some conditions on the variable group, which states that this group is in some sense similar to the infinite cyclic.

Let us give some examples. We call an equation

$$
w(G, t) \equiv \prod g_{i} t^{\varepsilon_{i}}=1, \quad \text { where } g_{i} \in G, \varepsilon_{i} \in \mathbb{Z}
$$

or a generalized equation

$$
w(G, T) \equiv \prod g_{i} t_{i}=1, \quad \text { where } g_{i} \in G, t_{i} \in T
$$

nontrivial if its left-hand side is not conjugate to a constant (i.e., to an element of the initial group $G$ ) in the free product $G *\langle t\rangle_{\infty}$ (respectively, in $G * T$ ).

Levin Conjecture [13]. Any nontrivial equation over a torsion-free group is solvable over this group.

This conjecture remains unproven, but it is well-known that it is equivalent to its generalized analogue.

This work was supported by the Russian Foundation for Basic Research, project no. 05-01-00895. 
Generalized Levin conjecture. Any nontrivial generalized equation with torsion-free variable group over a torsion-free group is solvable over this group.

Indeed, consider a torsion-free group $G_{1}$ containing the groups $G$ and $T$ as subgroups (e.g., $G_{1}=G \times T$ or $\left.G_{1}=G * T\right)$. Starting with a nontrivial generalized equation $\left(1^{\prime}\right)$ over $G$, we construct the nontrivial usual equation

$$
v\left(G_{1}, t\right) \equiv w\left(G_{1}, t^{-1} T t\right)=1
$$

over $G_{1}$. If the (usual) Levin conjecture is true, then this equation has a solution $\widetilde{t} \in \widetilde{G_{1}} \supseteq G_{1}$. But then the group $\widetilde{T}=\widetilde{t}^{-1} T \widetilde{t} \subseteq \widetilde{G}_{1}$ is a solution to the generalized equation $\left(1^{\prime}\right)$ over the group $G$. This proves the generalized Levin conjecture modulo the usual Levin conjecture.

Note that, in the generalized Levin conjecture, the variable group is required to be similar to the infinite cyclic in the sense that it istorsion-free.

The most known result related to the Levin conjectures is the following Brodskii-Howie theorem ([1], [10]). Recall, that a group is called locally indicable, if each of its nontrivial finitly generated subgroups admits an epimorphism onto the infinite cyclic.

Theorem B. Over a locally indicable group any nontrivial equation is solvable.

The generalized analogue of this fact is the following theorem (which was actually proved in [1]).

Theorem B'. Over a locally indicable group any nontrivial generalized equation with locally indicable variable group is solvable.

To prove the equivalence of these two theorems, it is sufficient to repeat word-by-word the arguments about the usual and generalized Levin conjecture; one should only replace "torsion-free" by "locally indicable".

Note that, in the Brodskii theorem, the variable group is required to be similar to the infinite cyclic in the sense of being locally indicable.

We can give many examples of such trivial generalizations. However, it is not so easy to generalize theorems related to another well-known conjecture. Recall, that equation (1) is called nonsingular if the exponent sum of $t$ in $w(G, t)$ is nonzero; if $\sum \varepsilon_{i}= \pm 1$, then the equation is called unimodular.

Kervaire-Laudenbach conjecture (see [3]). Any unimodular equation over any group is solvable over this group.

Sometimes (see, e.g., [5], [10]) the term "Kervaire-Laudenbach conjecture" is used for the hypothesis about solvability of any nonsingular equation (or even system of equations) over any group. At present, no version of this conjecture is proven or disproven.

Trying to formulate a generalized analogue of the Kervaire-Laudenbach conjecture or any known related facts, we meet the problem: What should we do with the condition of unimodularity (nonsingularity)?

In this paper we prove a generalized analogue of the following theorem.

Theorem 1 ([12], see also [8]). Any unimodular equation over a torsion-free group is solvable over it.

The generalized analogue we are going to prove is as follows.

Theorem 1'. Any unimodular generalized equation over a torsion-free group is solvable over it.

Here, unimodularity of a generalized equation is understood in the sense of the following definition that looks not quite natural, but does work and, of course, coincides with the usual unimodularity when the variable group is infinite cyclic.

Definition 1. Generalized equation $\left(1^{\prime}\right)$ is called unimodular if

1) the order of $\prod t_{i}$ in the group $T$ is infinite;

2) $\left\langle\prod t_{i}\right\rangle \triangleleft T$;

3) $T /\left\langle\prod t_{i}\right\rangle$ is a group with the strong unique product property.

Recall that a group $H$ is called a UP-group or a group with the unique product property if the product $X Y$ of any two finite nonempty subsets $X, Y \subseteq H$ contains at least one element, which uniquely decomposes 
into the product of an element from $X$ and an element from $Y$. It is known that the group ring of a UP-group contains no zero divisors. Some time ago, there was the conjecture that any torsion-free group is UP (the converse is, obviously, true). If this conjecture were true, then the Kaplansky problem on zero divisors in group rings would be solved. However, it turned out that there exist counterexamples ([17], [16]).

We say that a group $H$ has the strong unique product property if the product $X Y$ of any two finite nonempty subsets $X, Y \subseteq H$ such that $|Y| \geqslant 2$ contains at least two uniquely decomposable elements $x_{1} y_{1}$ and $x_{2} y_{2}$ such that $x_{1}, x_{2} \in X, y_{1}, y_{2} \in Y$ and $y_{1} \neq y_{2}$.

Note that the essence of this definition is the condition $y_{1} \neq y_{2}$, since according to a theorem of Strojnowski [19] the product of any two finite nonempty nonsingleton subsets of a UP-group always contains at least two uniquely decomposable elements.

As far as we know, all known examples of UP-groups has the strong UP-property. In particular, right orderable groups, locally indicable groups, diffuse (and weakly diffuse) groups in the sense of Bowditch have the strong UP property.

Note also that the strong unique product property follows from the following property $\mathrm{UP}_{4}$ : the product of any 4 nonempty finite subsets $A, B, C, D \subseteq H$ contains at least one element uniquely decomposable into a product $a b c d$, where $a \in A, b \in B, c \in C$ and $d \in D$. Indeed, if all uniquely decomposable elements $x y$ of the set $X Y$ have the same factor $y$, then the product of 4 subsets $X Y Y^{-1} X^{-1}$ contains no uniquely decomposable elements: the uniqueness decomposability of an element $u=x_{1} y_{1} y_{2}^{-1} x_{2}^{-1}$ implies that $y_{1}=y$ (otherwise $x_{1} y_{1}$ would have another decomposition $x_{1} y_{1}=x_{1}^{\prime} y_{1}^{\prime}$, and hence $u$ would have another decomposition $\left.u=x_{1}^{\prime} y_{1}^{\prime} y_{2}^{-1} x_{2}^{-1}\right)$; the similar arguments show that $y_{2}$ shoud be equal to $y$; but then $u$ has two different decompositions $u=x_{1} y y^{-1} x_{2}^{-1}=x_{1} y^{\prime}\left(y^{\prime}\right)^{-1} x_{2}^{-1}$, where $y^{\prime} \in Y$ is an arbitrary element other than $y$.

Theorem 1' implies the following multivariable analogue of Theorem 1.

Corollary. An equation

$$
g_{1} x_{j_{1}}^{\varepsilon_{1}} g_{2} x_{j_{2}}^{\varepsilon_{2}} \ldots g_{n} x_{j_{n}}^{\varepsilon_{n}}=1
$$

over a torsion-free group $G$ with variables $x_{1}, x_{2}, \ldots$ is solvable over $G$ if $\prod x_{j_{i}}^{\varepsilon_{i}}$ is not a proper power in the free group $F\left(x_{1}, x_{2}, \ldots\right)$.

Proof. Consider the group

$$
T=\left\langle x_{1}, x_{2}, \ldots \mid\left[x_{1}, \prod x_{j_{i}}^{\varepsilon_{i}}\right]=1,\left[x_{2}, \prod x_{j_{i}}^{\varepsilon_{i}}\right]=1, \ldots\right\rangle .
$$

This group is a universal central extension of the one-relator group $T_{1}=\left\langle x_{1}, x_{2}, \ldots \mid \prod x_{j_{i}}^{\varepsilon_{i}}=1\right\rangle$. It is well-known that, if $\prod x_{j_{i}}^{\varepsilon_{i}}$ is not a proper power in the free group $F\left(x_{1}, x_{2}, \ldots\right)$, then $T_{1}$ is locally indicable ([1]) and, hence, $T_{1}$ is a UP-group. The element $\prod x_{j_{i}}^{\varepsilon_{i}}$ have infinite order in the group $T$ (see [3]). Thus, equation $(* *)$ can be considered as a unimodular generalized equation with variable group $T$. Therefore, this equation is solvable by virtue of Theorem $1^{\prime}$ and the corollary is proven.

Theorem $1^{\prime}$ will be obtained as a special case of the following statement.

Main theorem. If any unimodular equation over any free power of a group $G$ is Magnusian, then any unimodular generalized equation over $G$ is solvable over $G$.

We say that equation (1) over a group $G$ is magnusian* if it is solvable over $G$ and, for any free factor $H$ of the group $G$ such that equation (1) is not an equation over it (in the sense, that $w$ is not conjugate in $G *\langle t\rangle$ to an element of the group $H *\langle t\rangle)$, some overgroup contains a solution $\widetilde{t}$ transcendental over $H$, i.e., such that $\langle H, \widetilde{t}\rangle=H *\langle\widetilde{t}\rangle_{\infty}$.

Theorem $1^{\prime}$ is a direct corollary of the main theorem and the following lemma, which is of independent interest.

Lemma 1. Any unimodular equation over a torsion-free group is magnusian.

Actually, the main theorem asserts nothing more than the validity of Theorem $1^{\prime}$, because, obviously, over a group with torsion there exists a nonmagnusian unimodular equation (e.g., the equation $t=g$, where $g$ is a nontrivial element of finite order).

* We mean Magnus's Freiheitssatz [15] (see also [3]), which we can formulate as follows: Any equation over a free group is magnusian. 
The notation which we use is mainly standard. Note only that if $x$ and $y$ are elements of a group, and $X$ is a subset of this group, then $x^{y}$ means $y^{-1} x y$, commutator $[x, y]$ is understood as $x^{-1} y^{-1} x y$, and the symbols $\langle X\rangle$ and $\langle\langle X\rangle\rangle$ denote, respectively, the subgroup, generated by the set $X$ and the normal subgroup, generated by the set $X$. In addition, if $A$ and $B$ are isomorphic subgroups of a group and $\varphi: A \rightarrow B$ is an isomorphism, then the system of relations $\left\{a^{x}=a^{\varphi} \mid a \in A\right\}$, which often arises in our consideration, will be writen in the reduced form $A^{x}=B$ if it is clear what isomorphism is meant.

\section{Proof of the main theorem}

Put $t=\prod t_{i}$. Let us decompose $T$ into the union of cosets:

$$
T=\coprod_{x \in T /\langle t\rangle} c_{x}\langle t\rangle, \quad \text { where } c_{1}=1 .
$$

We rewrite generalized equation $\left(1^{\prime}\right)$ in the form

$$
t \prod_{i} g_{i}^{c_{x_{i}} t^{k_{i}}}=1
$$

Let $X_{1}=\left\{x_{i}\right\}$ be the set of all $x \in T /\langle t\rangle$ occuring in the irreducible form of equation (2). For each $x \in T /\langle t\rangle$, let $G^{\left(c_{x}\right)}$ denote an isomorphic copy of the group $G$ assuming that the isomorphism maps $g \in G$ to $g^{\left(c_{x}\right)} \in G^{\left(c_{x}\right)}$.

Put

$$
H_{1}=\underset{y \in X_{1}}{*} G^{\left(c_{y}\right)}
$$

and consider the equation

$$
t \prod_{i} g_{i}^{\left(c_{x_{i}}\right) t^{k_{i}}}=1
$$

(with variable $t$ ) over the group $H_{1}$.

Let us conjugate equation (2) by an element $x \in T /\langle t\rangle$ (we mean that we conjugate by an element of $T$, but conjugation by $t$ gives an equation equivalent to the initial one). We obtain the equation

$$
t^{\varepsilon_{x}} \prod_{i} g_{i}{ }^{c_{x_{i} x} t^{l_{i}(x)}}=1
$$

where $\varepsilon_{x}= \pm 1$ depending on whether or not $x$ and $t$ commute, and the integers $l_{i}(x)$ are uniquely determined by the equalities $c_{x_{i}} t^{k_{i}} c_{x}=c_{x_{i} x} t^{l_{i}(x)}$. Similarly, we put $X_{x}=X_{1} x=\left\{x_{i} x\right\}$ and write the equation

$$
w_{x}(t) \equiv t^{\varepsilon_{x}} \prod_{i} g_{i}{ }^{\left(c_{x_{i} x}\right) t^{l_{i}(x)}}=1
$$

(with variable $t$ ) over the group

$$
H_{x}=\underset{y \in X_{x}}{*} G^{\left(c_{y}\right)}
$$

According to Theorem 1, this unimodular equation over a torsion-free group has a solution $\widetilde{t} \in \widetilde{H_{x}} \supseteq H_{x}$.

Certainly, we can assume that

$$
\widetilde{H_{x}}=\left\langle H_{x}, \widetilde{t} \mid w_{x}(\widetilde{t})=1\right\rangle .
$$

Put

$$
K=\left\langle\underset{x \in T /\langle t\rangle}{*} G^{\left(c_{x}\right)}, \widetilde{t} \mid\left\{w_{y}(\widetilde{t})=1 \mid y \in T /\langle t\rangle\right\}\right\rangle .
$$

Let us prove that the natural mapping $G \simeq G^{(1)} \rightarrow K$ is injective. To do this, it is sufficient to show that, for any finite set $Y \subseteq T /\langle t\rangle$ such that $X_{1} Y \ni 1$, the natural mapping

$$
G \simeq G^{(1)} \rightarrow K_{Y}=\left\langle\underset{x \in X_{1} Y}{*} G^{\left(c_{x}\right)}, \tilde{t} \mid\left\{w_{y}(\widetilde{t})=1 \mid y \in Y\right\}\right\rangle
$$

is injective. This is an obvious corollary of the following lemma. 
Lemma 2. Suppose that $Y$ is a finite subset of the group $T /\langle t\rangle, z \in T /\langle t\rangle, k=\left|X_{1}\right|$, and $y_{1}, \ldots, y_{k-1}$ are different elements of $X_{z} \cap\left(X_{1} Y\right)$. Then in the group $K_{Y}$ we have

$$
\left\langle\widetilde{t}, G^{\left(c_{y_{1}}\right)}, \ldots, G^{\left(c_{y_{k-1}}\right)}\right\rangle=\langle\widetilde{t}\rangle_{\infty} * G^{\left(c_{y_{1}}\right)} * \ldots * G^{\left(c_{y_{k-1}}\right)}
$$

(Note that the element $z$ may lie either inside or outside $Y$.)

Proof. Induction on the cardinality of the set $Y$. If $Y=\{u\}$, then the assertion of the lemma follows from that the unimodular equation $w_{u}(t)=1$ over the group $H_{u}$ is magnusian (Lemma 1 ), because $K_{Y}=K_{\{u\}}=\widetilde{H_{u}}$.

If $|Y|>1$, then the strong UP property of the group $T /\langle t\rangle$ implies that there exists $y \in Y \backslash\{z\}$ such that $X_{y}=X_{1} y \nsubseteq X_{1} \cdot(\{z\} \cup Y \backslash\{y\})$, therefore $\left|\left(X_{1} \cdot(\{z\} \cup Y \backslash\{y\})\right) \cap X_{y}\right|<k$; hence, in the group $K_{Y \backslash\{y\}}$, by the induction hypothesis we have

$$
\left\langle\widetilde{t},\left\{G^{\left(c_{x}\right)} ; x \in\left(X_{1} \cdot(Y \backslash\{y\})\right) \cap X_{y}\right\}\right\rangle=\langle\widetilde{t}\rangle_{\infty} *\left(\begin{array}{c}
\underset{\left(X_{1} \cdot(Y \backslash\{y\})\right) \cap X_{y}}{*} G^{\left(c_{x}\right)}
\end{array}\right) .
$$

Therefore, in the group

$$
N=K_{Y \backslash\{y\}} *\left(\begin{array}{c}
* \\
x \in\left(X_{z} \cap X_{y}\right) \backslash\left(X_{1}(Y \backslash\{y\})\right)
\end{array} G^{\left(c_{x}\right)}\right)
$$

we have

$$
M=\left\langle\widetilde{t},\left\{G^{\left(c_{x}\right)} ; x \in\left(X_{1} \cdot(\{z\} \cup Y \backslash\{y\})\right) \cap X_{y}\right\}\right\rangle=\langle\widetilde{t}\rangle_{\infty} *\left(\underset{x \in\left(X_{1} \cdot(\{z\} \cup Y \backslash\{y\})\right) \cap X_{y}}{*} G^{\left(c_{x}\right)}\right) .
$$

The same decomposition of the group $M$ is valid in $\widetilde{H}_{y}$ by Lemma 1 . Thus, we obtain a correct decomposition into the amalgamated product

$$
K_{Y}=\underset{M}{*} \underset{H_{y}}{ }
$$

The subgroup $\left\langle\widetilde{t}, G^{\left(c_{y_{1}}\right)}, \ldots, G^{\left(c_{y_{k-1}}\right)}\right\rangle$ lies in $N$ and the assertion of the lemma follows from the induction hypothesis. Lemma 2 , and the injectivity of the natural mapping $G \simeq G^{(1)} \rightarrow K$ is proven.

The group $T$ acts on $K$ by the rule

$$
\widetilde{t}^{y}=\widetilde{t}^{\varepsilon_{y}}, \quad\left(g^{\left(c_{x}\right)}\right)^{y}=\left(g^{\left(c_{f}\right)}\right)^{\widetilde{t}^{k}}
$$

where $y \in T$ is an arbitrary element, and $f \in T /\langle t\rangle$ and $k \in \mathbb{Z}$ are determined from the equalities $c_{x} y=c_{f} t^{k}$. Obviously, this is a well defined action by automorphisms.

Take the corresponding semidirect product $T \nless K$ and consider its quotient group by the normal cyclic subgroup $\left\langle\widetilde{t} t^{-1}\right\rangle$. This is the sought group containing a solution:

$$
\widetilde{G}=(T<K) /\left\langle\widetilde{t t}^{-1}\right\rangle .
$$

Indeed, $G$ is embedded into $\widetilde{G}$ as subgroup: $G=G^{(1)} \subseteq K \subseteq \widetilde{G}$. According to the definition of action (3), we have

$$
G^{\left(c_{x}\right)}=G^{c_{x}}
$$

hence, the relation $w_{1}(\widetilde{t})=1$ holds in $K$ and equality $t=\widetilde{t}$ in $\widetilde{G}$ give relation (2). Thus, the group $T$ considered as a subgroup of $\widetilde{G}$ is a solution to equation (2). The main theorem is proven. 


\section{Proof of Lemma 1}

First, note one simple fact.

Lemma 3. Suppose that $A$ is a nontrivial subgroup of a group $B$ and $b \in B$. Then $b$ is transcendental over $A$ if and only if

$$
\left\langle\left\{A^{b^{i}} \mid i \in \mathbb{Z}\right\}\right\rangle=\underset{i \in \mathbb{Z}}{*} A^{b^{i}} .
$$

Proof. The "only if" part is obvious. The "if" part follows from the fact that, if $u \in A *\langle b\rangle_{\infty}$ is a nontrivial relation between $A$ and $b$ in the group $B$ and $a \in A \backslash\{1\}$, then $[a, u]$ is a nontrivial relation between the groups $A^{b^{i}}$. Lemma 3 is proven.

Throughout this section, we suppose that $G=H * K$, equation (1) is an unimodular equation over $G$, and $w$ does not belong to a subgroup conjugate to $H *\langle t\rangle$ in the group $G *\langle t\rangle_{\infty}$. In addition, we put $U=G *\langle t\rangle_{\infty} /\langle\langle w\rangle\rangle$ (this is the so caled universal solution group). We have to prove that equation (1) has a solution transcendental over $H$, or, equivalently, the element $t \in U$ is transcendental over $H$.

Lemma 4. If

$$
\left\langle\left\{H^{t^{i}} \mid i \in \mathbb{Z}\right\}\right\rangle=\underset{i \in \mathbb{Z}}{*} H^{t^{i}}
$$

in $U$, then $t \in U$ is a solution to equation (1) transcendental over $H$.

Proof. In the case when the group $H$ is nontrivial, the assertion immediately follows from Lemma 3 . If $H=\{1\}$, then the lemma asserts only that the element $t \in U$ has infinite order (if $w$ is not conjugate to $\left.t^{ \pm 1}\right)$; this fact is observed in [6]. Lemma 4 is proven.

Put

$$
\bar{H}=\left(\underset{i \in \mathbb{Z}}{*} H_{i}\right), \quad \bar{G}=\bar{H} * K,
$$

where $H_{i}$ are isomorphic copies of the group $H$. Identifying the group $G$ with the subgroup $N_{0} * K$ of $\bar{G}$ consider the system of equations over $\bar{G}$ consisting of initial equation (1) and auxiliary equations

$$
\left\{\begin{aligned}
w(t) & =1, \\
t^{-1} H_{i} t & =H_{i+1}, \quad i \in \mathbb{Z} .
\end{aligned}\right.
$$

Using the obvious transformations this system can be rewriten in the form

$$
\left\{\begin{aligned}
w_{1}(t) & =1, \\
t^{-1} H_{i} t & =H_{i+1}, \quad i \in \mathbb{Z},
\end{aligned}\right.
$$

where $w_{1}$ contains no fragments of the form $t^{-1} \bar{h} t$ and $t \bar{h} t^{-1}$ with $\bar{h} \in \bar{H}$.

If the length of $w_{1}(t)$ is 1 , then the first equation of this system can be rewriten in the form $t=u$, where $u \in \bar{G}$, and the entire system can be rewriten in the form

$$
\left\{u^{-1} H_{i} u=H_{i+1}, \quad i \in \mathbb{Z}\right\} .
$$

The natural mapping of the group $\bar{N}$ into the group $\overline{\bar{G}}=\left\langle\bar{G} \mid\left\{u^{-1} H_{i} u=H_{i+1}, \quad i \in \mathbb{Z}\right\}\right\rangle$ is injective by the following theorem proven in [12] (see also [8]).

Theorem. Suppose that $A$ and $B$ are torsion-free groups, $v \in(A * B) \backslash A$, and $\varphi$ is an automorphism of $A$. Then the natural mappings

$$
A \rightarrow\left\langle A * B \mid\left\{a^{v}=a^{\varphi} \mid a \in A\right\}\right\rangle \leftarrow B
$$

are injective.

The element $t$ of the HNN-extension $\widetilde{G}=\left\langle\overline{\bar{G}}, t \mid H_{i}^{t}=H_{i+1} i \in \mathbb{Z}\right\rangle$ is obviously a solution to equation (1) over $G=H_{0} * K$. Clearly, it is transcendental over $H=H_{0}$ by Lemma 4, because relation (4) is fulfilled in $\widetilde{G}$ and, hence, in $U$. (Actually, it is easy to observe that $\widetilde{G} \simeq U$.) 
Now, consider the case when the length (that is, the number of occurrences of $t^{ \pm 1}$ ) of the word $w_{1}$ is greater than one. Consider the following subgroups of $G *\langle t\rangle_{\infty}: K_{i}=t^{-i} K t^{i}, H_{i}=t^{-i} H t^{i}$,

$$
\bar{H}=\underset{i=-\infty}{\stackrel{\infty}{*}} H_{i}, \quad K^{(m)}=\underset{i=0}{\stackrel{m}{*}} K_{i} \quad \text { and } \quad G^{(m)}=\bar{H} * K^{(m)} .
$$

Consider all possible expressions of equation (1) in the form

$$
c t \prod_{i=1}^{n} b_{i} t^{-1} a_{i} t=1, \quad \text { where } a_{i}, b_{i}, c \in G^{(m)} .
$$

Among all such expressions we choose those in which $m$ is minimal; after that, from all expressions with minimal $m$ we choose an expression with minimal $n$. For such a minimal expression (6), we have:

1) $n \geqslant 1$ (i.e., the length of this expression is strictly greater than one);

2) $a_{i} \notin G^{(m-1)}=\bar{H} * K_{0} * \ldots * K_{m-1}$ and $b_{i} \notin\left(G^{(m-1)}\right)^{t}=\bar{H} * K_{1} * \ldots * K_{m}$;

3) $a_{i}$ are transcendental over $G^{(m-1)}$, and $b_{i}$ are transcendental over $\left(G^{(m-1)}\right)^{t}$.

The first property is ensured by the assumption that the length of $w_{1}$ is greater than one (in any expression of length 1 we have $m>0$; therefore, we can decrease $m$ by replacing all occurrences of elements of $K_{m}$ by fragments $t^{-1} g t$, where $g \in K_{m-1}$ ). The second property obviously follows from the minimality of $n$ and $m$. Property 3 ) is a corollary of property 2 ).

Now, suppose that the symbols $H_{i}$ and $K_{i}$ denote abstract isomorphic copies of the groups $H$ and $K$, and the groups $\bar{H}, K^{(m)}$ and $G^{(m)}$ are defined by formulae (5). Consider the following system of equations over the group $G^{(m)}$ :

$$
\left\{\begin{array}{l}
x^{-1} H_{i} x=H_{i+1}, \quad i \in \mathbb{Z}, \\
x^{-1} K_{i} x=K_{i+1}, \quad i \in\{0, \ldots m-1\} \\
c x \prod_{i=1}^{n} b_{i} x^{-1} a_{i} x=1 .
\end{array}\right.
$$

Clearly, any solution to this system over $G^{(m)}$ is a solution to equation (1) over $G$ transcendental over $H$ (by Lemma 4). To complete the proof of Lemma 1, we should only note that properties 1) and 3) of system (7) imply its solvability in virtue of the following theorem.

Theorem ([12], see also [8]). Suppose that $M$ and $N$ are isomorphic subgroups of a group $L, \varphi: M \rightarrow N$ is an isomorphism, $n \in \mathbb{N}, a_{1}, \ldots, a_{n}$ are elements of $L$ transcendental over $M, b_{1}, \ldots, b_{n}$ are elements of $L$ transcendental over $N$, and $c \in L$. Then the system of equations

$$
\left\{\begin{array}{l}
x^{-1} g x=g^{\varphi}, \quad g \in M, \\
c x \prod_{i=1}^{n} b_{i} x^{-1} a_{i} x=1
\end{array}\right.
$$

is solvable over $L$. 


\section{Concluding remarks}

The definition of the unimodularity of generalized equations (Definition 1) would look nicer, if condition 3) had the form

$\widetilde{3})$ the group $T /\left\langle\prod t_{i}\right\rangle$ is torsion-free.

We call generalized equation (1') satisfying conditions 1), 2) and $\widetilde{3}$ ) weakly unimodular.

Question 1. Is it true that any weakly unimodular generalized equation over a torsion-free group is solvable over it?

If we completly remove condition 3) from Definition 1, we shall obtain the notion of a nonsingular generalized equation.

Question 2. Is it true that any nonsingular generalized equation over a torsion-free group is solvable over it?

The answer to this question is unknown even for usual equations, in the generalized situation it might be easier to construct a counterexample.

Is it possible to prove generalized analogues of others known facts? for example, the GerstenhaberRothaus theorem ([9], see also [3])?

Question 3. Is it true that any (weakly) unimodular (or even any nonsingular) generalized equation over a finite group is solvable over it?

It is easy to see that the Levin conjecture implies that any solvable equation over a torsion-free group is magnusian. Let us state the following conditional question.

Question 4. Is the conjecture that any solvable equation over a torsion-free group is magnusian equivalent to the Levin conjecture?

It is easy to see that the answer to the generalized version of this question is positive.

\section{REFERENCES}

[1] Brodskii S. D. Equations over groups and one relator groups (in Russian) // Sib. Mat. Zh. 1984. T.25. 2. S.84-103.

[2] Klyachko Ant. A., Prishchepov M. I. The descent method for equations over groups (in Russian) // Vestn. MSU: Mat., Mech. 1995. 4. S.90-93.

[3] R. C. Lyndon and P. E. Schupp, Combinatorial group theory, Springer-Verlag, 1977.

[4] Clifford A. Nonamenable type K equations over groups // Glasgow Math. J. 2003. V.45. P.389-400.

[5] Clifford A., Goldstein R. Z. Equations with torsion-free coefficients // Proc. Edinburgh Math. Soc. 2000. V.43. P.295-307.

[6] Cohen M. M., Rourke. C. The surjectivity problem for one-generator, one-relator extensions of torsionfree groups // Geometry \& Topology. 2001. V.5. P.127-142.

[7] Edjvet M., Howie J. The solution of length four equations over groups // Trans. Amer. Math. Soc. 1991. V.326. P.345-369.

[8] Fenn R., Rourke C. Klyachko's methods and the solution of equations over torsion-free groups // L'Enseignment Mathématique. 1996. T.42. P.49-74.

[9] Gerstenhaber M., Rothaus O. S. The solution of sets of equations in groups // Proc. Nat. Acad. Sci. USA. 1962. V.48 P.1531-1533.

[10] Howie J. On pairs of 2-complexes and systems of equations over groups // J. Reine Angew Math. 1981. V.324. P.165-174.

[11] Ivanov S. V., Klyachko Ant. A. Solving equations of length at most six over torsion-free groups // J. Group Theory. 2000. V.3. P.329-337.

[12] Klyachko Ant. A. A funny property of a sphere and equations over groups // Comm. Algebra. 1993. V.21. P.2555-2575.

[13] Levin F. Solutions of equations over groups // Bull. Amer. Math. Soc. 1962. V.68. P.603-604.

[14] Lyndon R. C. Equations in groups // Bol. Soc. Bras. Math. 1980. V.11. 1. P.79-102. 
[15] Magnus W. Über diskontinuierliche Gruppen mit einer definierenden Relation (Der Freiheitssatz). // J. Reine Angew Math. 1930. V.163. P.141-165.

[16] Promyslow S. D. A simple example of a torsion free nonunique product group // Bull. London Math. Soc. 1988. V.20. P.302-304.

[17] Rips E., Segev Y. Torsion free groups without unique product property // J. Algebra 1987. V.108. P.116-126.

[18] Stallings J. R. A graph-theoretic lemma and group embeddings // Combinatorial group theory and topology (ed. Gersten S. M, Stallings J. R.). Annals of Mathematical Studies. 1987. V.111. P.145-155.

[19] Strojnowski A. A note on u.p. groups // Comm. Algebra 1980. V.8. P.231-234. 